\title{
Comparison of Cervical Epidural Anesthesia and General Anesthesia for Thyroid Surgery
}

\author{
${ }^{1}$ Shamna Mohammed, ${ }^{2}$ UP Santosh
}

\section{ABSTRACT}

Introduction: National trends are showing statistically significant increase in the thyroid surgeries being performed by otorhinolaryngologist surgeons. There is a rising interest among the otorhinolaryngology surgeons subspecializing in head and neck surgeries to increase the horizons in the advancements in thyroid surgeries. Cervical epidural anesthesia (CEA) for thyroid surgeries is gaining popularity over general anesthesia (GA) for the various advantages it offers.

Objective: This prospective study was designed to evaluate the effectiveness and ease of thyroidectomy under CEA as compared to GA.

Materials and methods: A prospective study of total of 30 patients undergoing thyroid surgery from two teaching hospitals. They were randomly allotted into CEA and GA groups of 15 members each.

Results: In the CEA group, postoperative comfort and economic feasibility were found to be better compared to GA group. The blood volume loss was lesser in the CEA group. The CEA group also offered the added advantage of vocal cord monitoring by voice contact during the surgery. Both the CEA and GA group maintained hemodynamic stability. There were no complications in both the groups.

Conclusion: Even though thyroid surgery is currently being performed mostly under GA, CEA must be considered for thyroid surgeries routinely. CEA offers a number of advantages of GA in selected cases. This study has proved that CEA has distinct advantages over GA in thyroidectomies. Considering the ease and effectiveness of CEA, it is as good as GA, if not better, even in routine thyroidectomies.

Keywords: Cervical epidural anesthesia, General anesthesia, Thyroidectomy.

\section{Source of support: Nil}

Conflict of interest: None

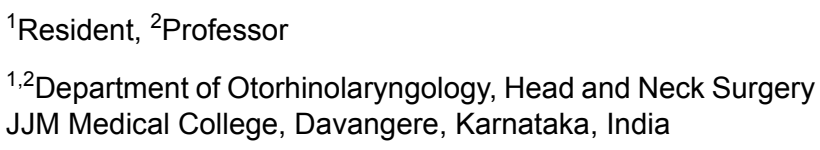

1,2Department of Otorhinolaryngology, Head and Neck Surgery JJM Medical College, Davangere, Karnataka, India

Corresponding Author: UP Santosh, Professor, Department of Otorhinolaryngology, Head and Neck Surgery, JJM Medical College, Davangere, Karnataka, India, Phone: +91-9845155223 e-mail: drsantoshup@gmail.com

\section{INTRODUCTION}

National trends are showing statistically significant increase in the thyroid surgeries being performed by otorhinolaryngologist surgeons. ${ }^{1}$ There is a rising interest among the otorhinolaryngology surgeons subspecializing in head and neck surgeries to increase the horizons in the advancements in thyroid surgeries. Cervical epidural anesthesia (CEA) for thyroid surgeries is gaining popularity over general anesthesia (GA) for the various advantages it offers.

Cervical epidural anesthesia was first popular by Degliotti et al in 1933. CEA for thyroid surgeries with anticipated difficult endotracheal intubation was encouraged by Khanna and Singh. ${ }^{2}$ Kook et al, ${ }^{3}$ recommended thyroid surgery under CEA for large thyroid tumors. They pointed out the advantage of vocal cord monitoring during surgery under CEA. ${ }^{3}$

Thyroid surgery although performed routinely under GA should be considered for extremely large thyroids with anticipated difficult endotracheal intubation. It should also be considered in thyroid surgeries with oscillating thyroid profiles, reported by medical condition predisposing to complications under GA. ${ }^{2}$

\section{MATERIALS AND METHODS}

A total of 30 patients undergoing thyroid surgery from two teaching hospitals were randomly allocated into two groups of 15 each. Approval was taken from the ethical committee. All patients' consent was taken prior to procedure. All patients were subjected to relevant blood, urine, ultrasonography and fine-needle aspiration cytology investigations.

The first group underwent thyroid surgery under CEA and second group under GA. All surgeries were performed by the same surgeon. All patients were in euthyroid state preoperatively. All the thyroid surgeries done were performed by the same surgeon and supervised by senior staff from anesthesia department. The study was conducted from November 2011 to November 2013.

\section{Technique of CEA}

Patient is made to sit with the forehead resting against a table with the chin touching sternum. The spine of the $\mathrm{C} 7$ vertebra is identified. The skin and subcutaneous tissue in $\mathrm{C} 7$ and $\mathrm{T} 1$ 
intervertebral space is infiltrated with $1 \%$ lignocaine. An 18-gauge Tuohy needle is inserted in the C7-T1 interspace (Fig. 1) perpendicular to the skin.

An epidural catheter is guided through the Tuohy needle and advanced. Five centimeter of the catheter is left inside the space (Fig. 2). The patient is placed in supine position. A total of $10 \mathrm{ml}$ of $2 \%$ of lignocaine with 1:2,00,000 adrenaline is injected through the catheter.

At the end of surgery, $10 \mathrm{ml}$ solution of $8 \mathrm{ml} 0.125 \%$ bupivacaine and $2 \mathrm{ml}$ of $100 \mathrm{mg}$ tramadol is given 6th hourly on the 1 st day followed by 8 th hourly on the 2 nd day.

\section{Technique of GA}

Routine general anesthetic techniques were followed for all thyroidectomies under GA.

\section{RESULTS}

The CEA for thyroid surgeries (Figs 3 to 5) revealed the following findings. No patient reported pain from the procedure. The upper margin of sensory block was assessed

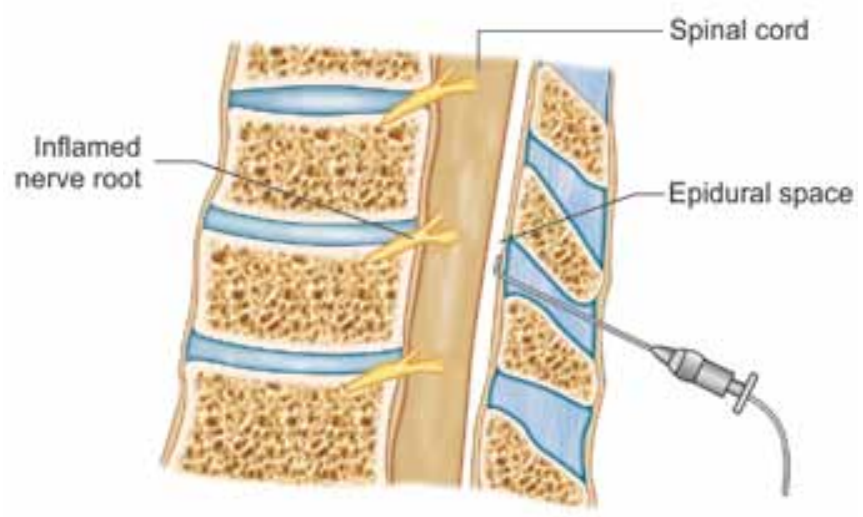

Fig.1: Epidural space

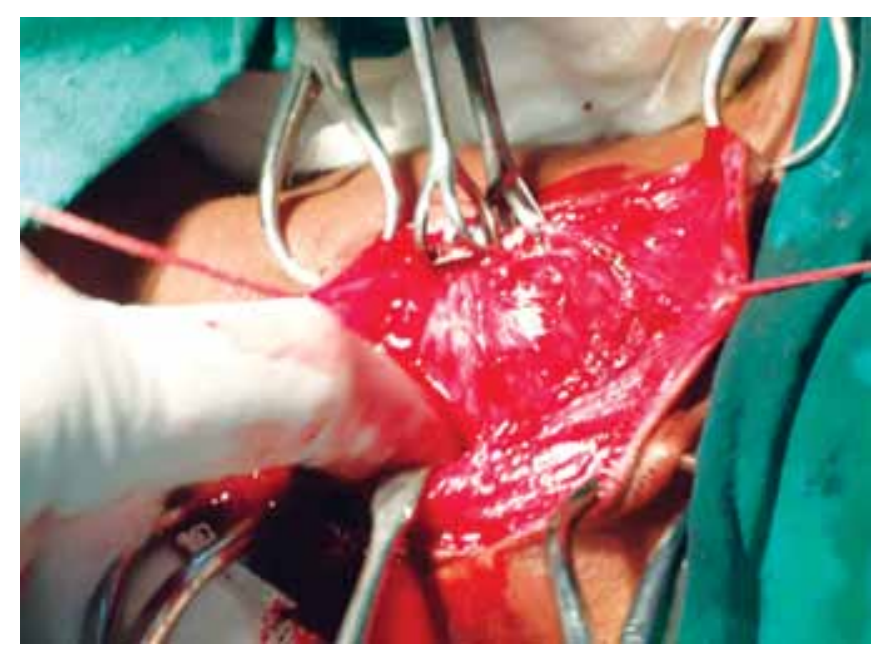

Fig. 3: Exposed thyroid gland under CEA in $\mathrm{C} 2$ dermatome in all patients and the median of the lower margin of sensory block was T2 (minimal extent C2-T1, maximal extent C2-T10). The patients were not in distress during surgery and did not complain of dyspnea during the procedure (Graphs 1 to 5). The patients were able to take orally in the immediate postoperative period. Pain, nausea, and vomiting were significantly reduced in this group. The patients were motivated to initiate ambulation early and were eager to be discharged as soon as the drain was removed. There was no case of accidental dura mater puncture or blood in the catheter.

The patient in the GA group suffered greater intensity of pain compared with the patients in the CEA group (Graph 6). There was increased incidence of vomiting. The patients in the GA group had increased duration of hospital stay (Graph 7). The patients in the CEA group had lower amount of blood loss (Graph 8).

\section{DISCUSSION}

The patients under CEA group were maintained in a state of conscious sedation or sedoanalgesia with verbal

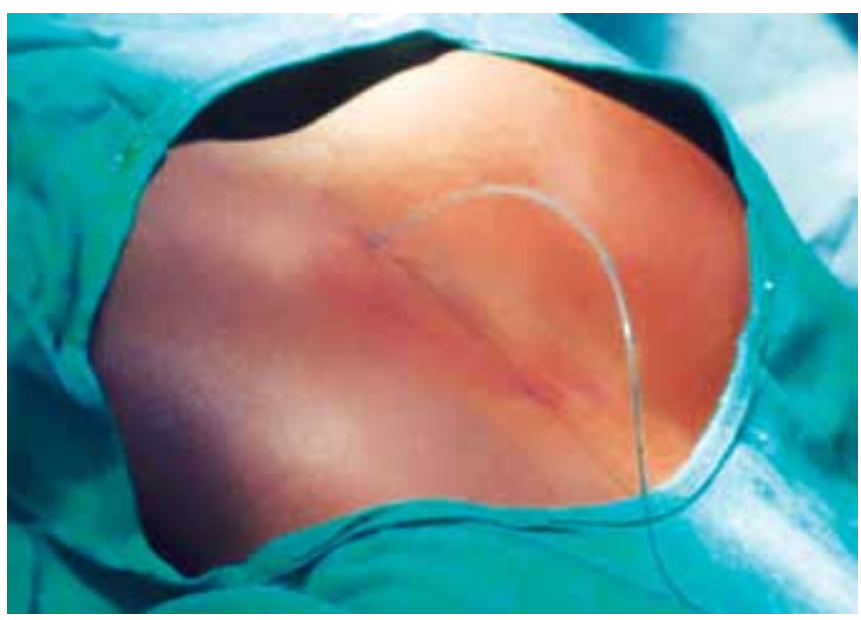

Fig. 2: Epidural catheter in place

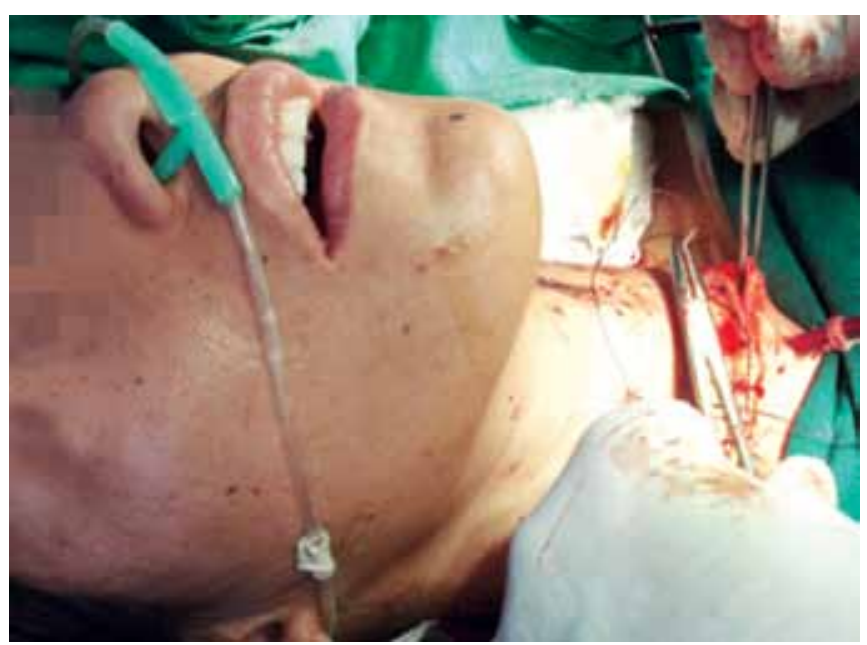

Fig. 4: Suturing under CEA 


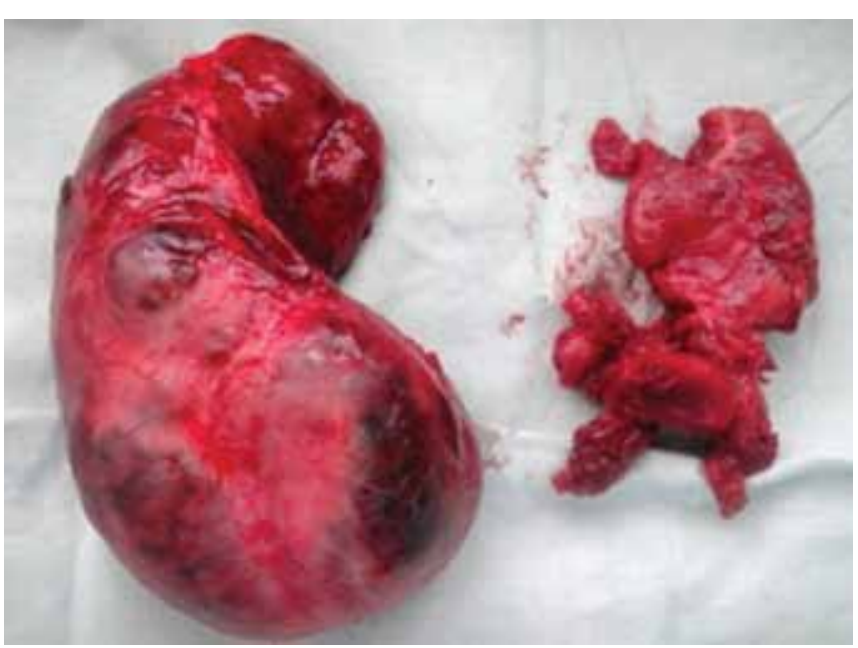

Fig. 5: A thyroid gland specimen excised under CEA

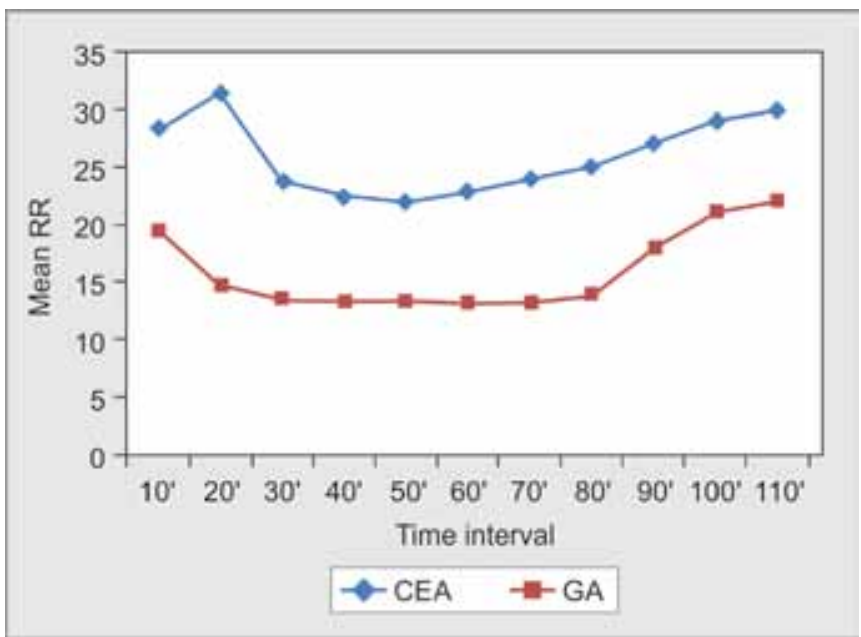

Graph 2: Variations in respiratory rate (RR) in two groups

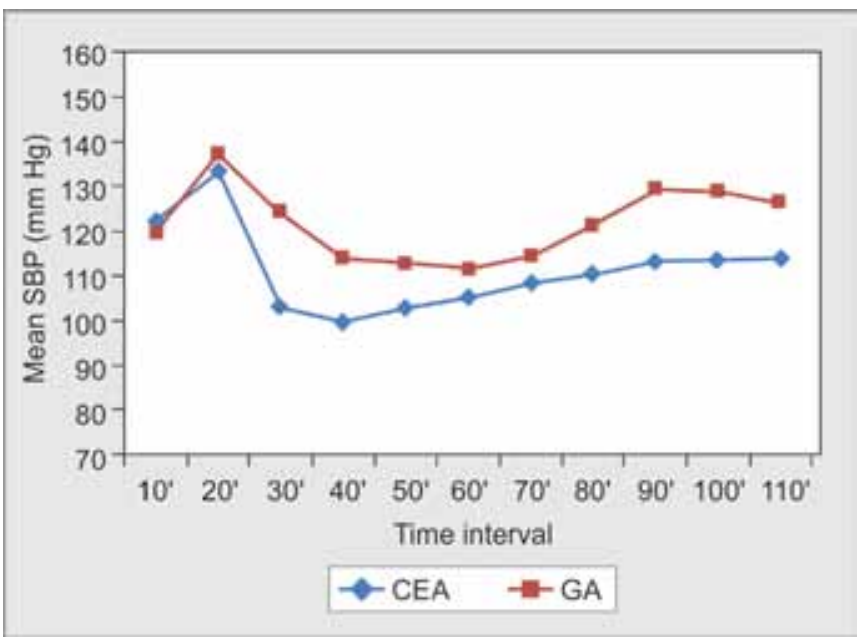

Graph 4: Variations in systolic blood pressure (SBP) in two groups

contact. This ensured that there was no trespass on the recurrent laryngeal nerve by voice monitoring. Other noted advantages of the CEA group over the GA group were as follows:

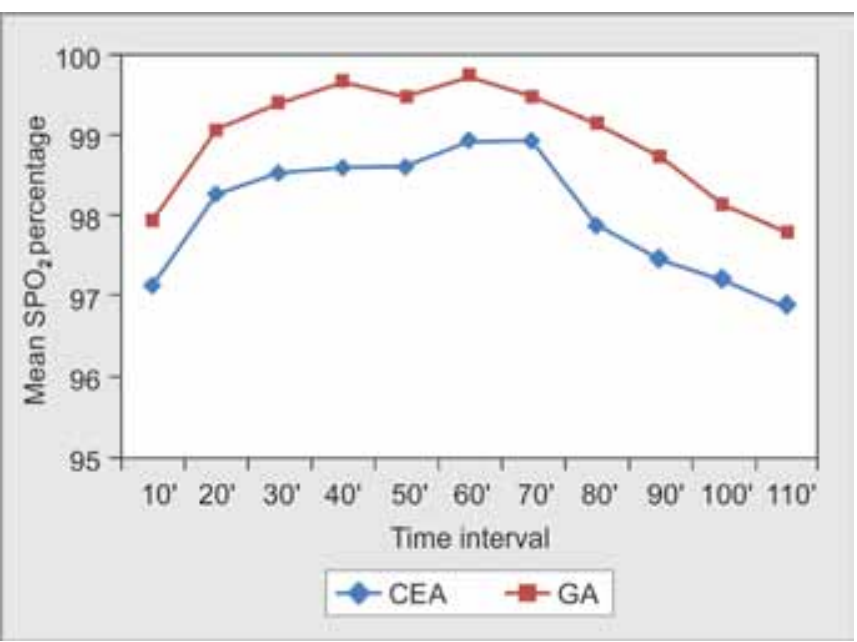

Graph 1: Variations in $\mathrm{SPO}_{2}$ in two groups

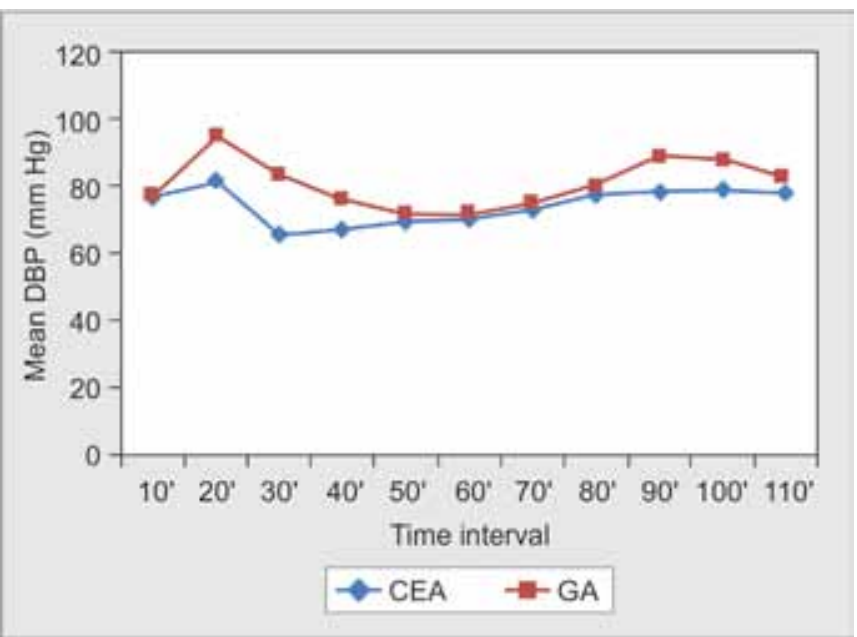

Graph 3: Variations in diastolic blood pressure (DBP) in two groups

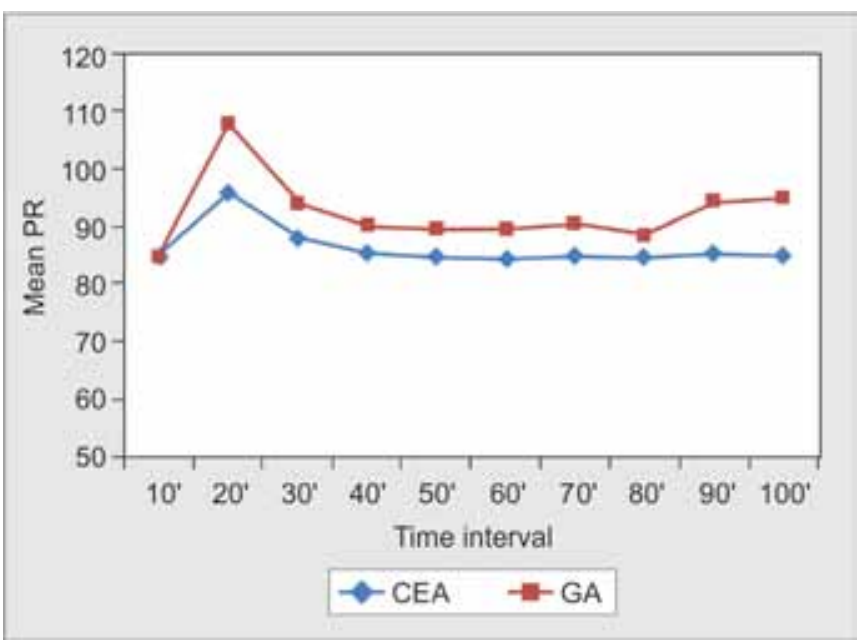

Graph 5: Variations in pulse rate (PR) in two groups

1. Less bleeding

2. Early ambulation

3. Better pain control

4. Little or no nausea. 


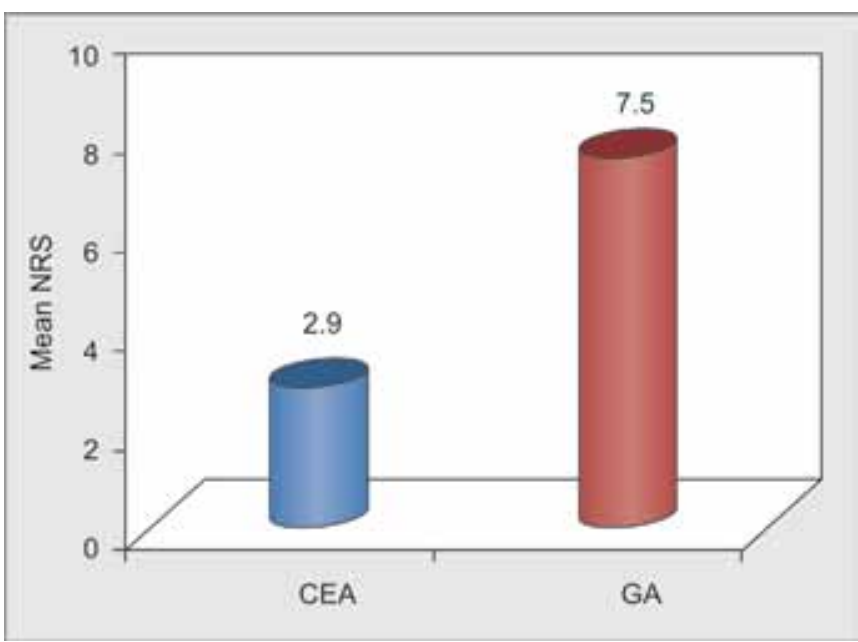

Graph 6: Postoperative pain incidence numerical rating score (NRS) in two groups

These findings coincided with those of Ahsan et al and Agrawal et al. ${ }^{4,5}$

Complications of CEA include its effects on respiratory function especially phrenic nerve controlled diaphragmatic movements and cardiovascular system. The other major concern with CEA has been its effects on heart rate and hemodynamic stability.

The most frequently reported side effects of CEA are hypotension and bradycardia. Other possible side effects are:

- Perforation of dura producing a total subarachnoid block.

- Traumatic hemorrhage caused by needle or catheter in the epidural space.

- Severe postspinal headache should dural puncture occur.

None of these complications were encountered in our study. Apart from this, it avoids the commonly associated GA risks such as nausea or vomiting, sore throat, headache, shivering, and delayed return to normal mental functioning.

\section{CONCLUSION}

Considering its advantages of stable hemodynamic and respiratory rate, control of pain extending into postoperative period and early ambulation with reduction in stress response, blood loss and postoperative morbidity.

We recommend CEA for thyroid surgeries as an alternative to GA for even routine thyroid surgeries.

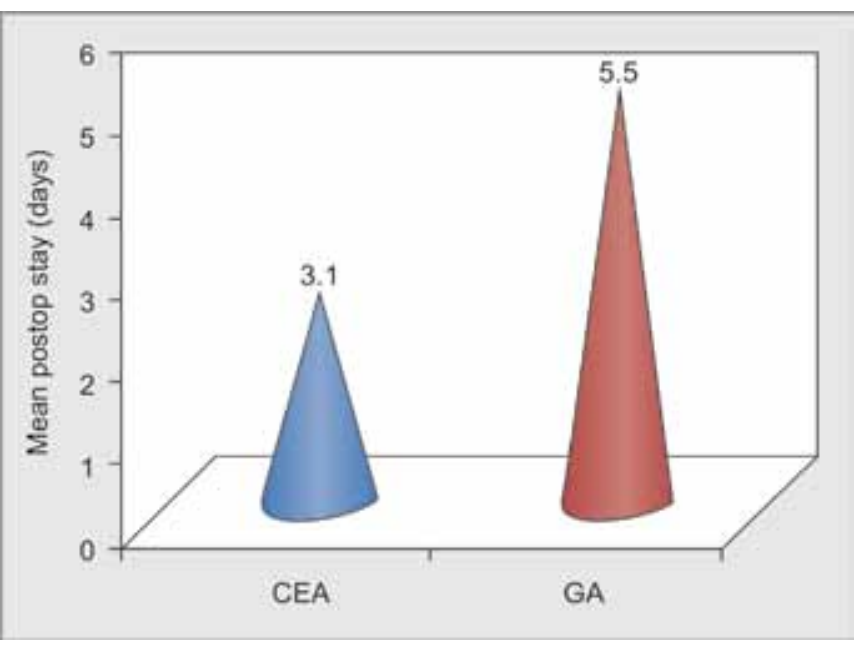

Graph 7: Postoperative hospital stay in two groups

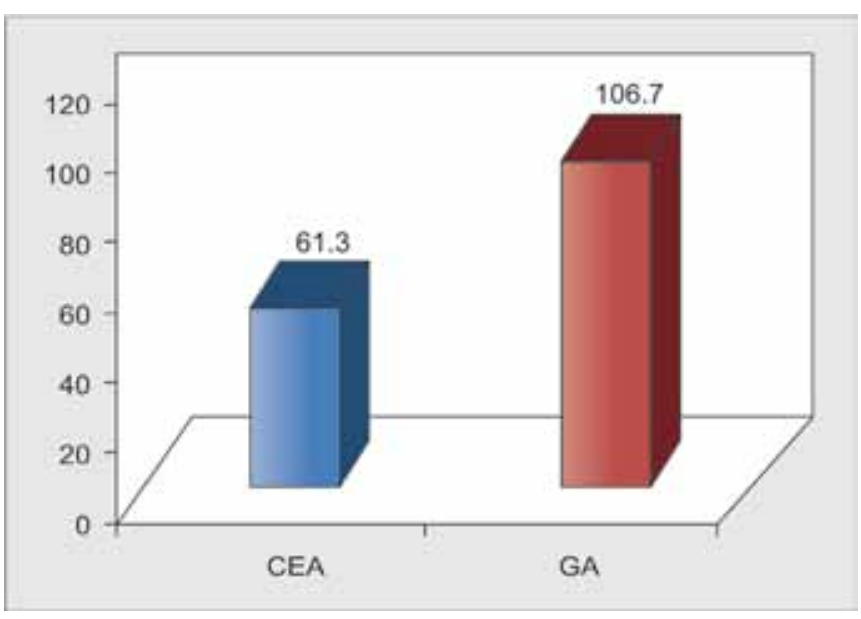

Graph 8: Blood loss (ml)

\section{REFERENCES}

1. Chambers KJ, Bhattacharyya N. The increasing role of otolaryngology in the management of surgical thyroid disorders. Laryngoscope 2013;123(12):3239-3242.

2. Khanna R, Singh DK. Cervical epidural anesthesia for thyroid surgery. Kathmandu Univ Med J (KUMJ) 2009;7(27):242-245.

3. Kook EY, Ahn YM, Lee C, Lee CS, Kim WT. Cervical epidural anesthesia for thyroidectomy in a patient with tracheal deviation: a case report. Korean J Anesthesiol 1999;37(1):159-163.

4. Ahsan SN, Faridi S. Cervical epidural anesthesia for subtotal thyroidectomy in a patient with aortic incompetence. J Pak Med Assoc 1998;48(9):281-283.

5. Agrawal M, Kang LS. Cervical epidural anesthesia for neck arm and upper thoracic surgery. J Anaesth Clin Pharmacol 2010;26(12):189-192. 